\title{
УДК 330.4
}

\author{
Р.В. Шамин ${ }^{1,2}$, Р.А. Чурсин ${ }^{2}$, А.Г. Шмелева ${ }^{1,2}$, Е.А. Галемина ${ }^{1}$, \\ С.Ю. Обыденнова ${ }^{3}$, Е.В. Фролагина ${ }^{3}$ \\ ОПТИМИЗАЦИЯ ИМПУЛЬСНЫХ ИНВЕСТИЦИЙ \\ НАУКОЕМКИХ ПРЕДПРИЯТИЙ
}

\author{
МИРЭА - Российский технологический университет ${ }^{1}$ \\ Российский университет дружбы народов ${ }^{2}$ \\ Нижегородский государственный технический университет им. Р.Е. Алексеева ${ }^{3}$
}

\begin{abstract}
В статье рассматривается имитационная модель, описывающая импульсные инвестиции для наукоемких предприятий. Под импульсными инвестициями понимается единовременное финансирование предприятия в заданные моменты времени. Предложена новая оптимизационная задача для количественных расчетов плана инвестиций для достижения заданных целевых показателей. Показано, что полученная экстремальная задача является некорректной по Тихонову, поэтому для построения вычислительных процедур необходимо использовать регуляризацию некорректных экстремальных задач по Тихонову. Для численного нахождения оптимального плана инвестиций используется метод роения частиц, который является эффективным при многомерной оптимизации для негладких функций. Представлен пример расчета оптимальной схемы импульсного финансирования в рамках предложенной экономико-математической модели.
\end{abstract}

Ключевые слова: имитационная модель, инвестиции, наукоемкие предприятия, оптимизационная задача, регуляризация некорректных экстремальных задач.

\section{Введение}

Проблема инвестиций в наукоемкие предприятия носит фундаментальный характер, поскольку финансирование НИР и НИОКР имеет собственную специфику [1]. В настоящей работе рассматриваются импульсные инвестиции, под которыми понимается единовременное финансирование предприятия в заданные моменты времени в течение длительного промежутка времени. В реальности эта модель хорошо отражает ситуацию, когда финансирование происходит раз в месяц или раз в год, и т.д. Предполагается, что в результате финансирования увеличивается результативность деятельности предприятия. Под результативностью мы понимаем некоторый интегральный количественный показатель. Разумеется, при отсутствии дальнейшего финансирования результативность должна снижаться. Основная проблема состоит в оптимальном распределении импульсного финансирования таким образом, чтобы обеспечить заданную результативность предприятия [2].

В работе предложена оригинальная математическая модель для описания процесса импульсного финансирования и построена оптимизационная задача для количественного расчета оптимальной схемы импульсных инвестиций. При этом следует учесть, что построенная оптимизационная задача является некорректной экстремальной задачей, поскольку решения этой задачи не зависит непрерывно от исходных данных [3]. Поэтому мы рассматриваем метод регуляризации этой некорректной задачи по Тихонову.

\section{Математические обозначения}

Рассмотрим экономико-математическую модель распределения инвестиций в наукоемкое предприятие. Ее целью является вычисление оптимального распределения инвестиций в рассматриваемое предприятие таким образом, чтобы поддерживать заданный уровень результативности инвестиций.

(C) Шамин Р.В., Чурсин Р.А., Шмелева А.Г., Галемина Е.А., Обыденнова С.Ю., Фролагина Е.В. 
Мы будем рассматривать некоторое предприятие $P$, управление которым будет состоять в распределении финансирования. При этом целью управления предприятием является обобщенный показатель результативности деятельности предприятия. Под результативностью можно понимать различные показатели, например, следующие:

- прибыль;

- уровень инновационности;

- конкурентоспособность и т.д.

Введем соответствующие обозначения. Будем рассматривать математическую модель с непрерывным временем, которое будем обозначать через $t$.

Распределение инвестиций мы будем описывать функцией $u(t)$, при этом будем считать, что эта функция представляет собой линейную комбинацию обобщенных функций, поскольку по своему экономическому смыслу - финансирование является импульсной функцией. Показатель результативности финансирования мы обозначим через $I(t)$. По экономическому смыслу - функции $u(t)$ и $I(t)$ являются неотрицательными.

\section{Динамическая модель}

Чтобы задать связь функций $u(t)$ и $I(t)$, необходимо ввести передаточную функцию $K(t)$, которая будет показывать результат вклада в функцию результативности $I(t)$ при импульсном воздействии функции $u(t)$ в момент времени $t=0$. Используя передаточную функцию, связь между функцией финансирования и результативности можно записать в виде интегрального уравнения (1)

$$
I(t)=\int_{0}^{t} K(t-s) u(s) d s .
$$

Передаточная функция имеет следующие свойства:

1) $K(t) \geq 0$.

2) $K(t)=0, t \leq 0$.

3) $K(t) \rightarrow 0, t \rightarrow \infty$.

Первое свойство показывает, что влияние финансирования на результативность не может быть отрицательным. Разумеется, в некоторых реальных ситуациях это свойство может нарушаться, но в нашей модели мы рассматриваем крупные инвестиции, поэтому считаем, что это влияние не может быть отрицательным.

Второе свойство показывает, что прошлое не зависит от будущего. Естественным образом финансирование влияет только на будущее, а не на прошлое.

Третье свойство отражает тот факт, что любые инвестиции имеют конечное время действия на результативность, и с течением времени их влияние нивелируется.

Уравнение (1) представляет собой линейное интегральное уравнение первого рода.

\section{Импульсные инвестиции}

Как мы уже отмечали, в экономических ситуациях мы должны рассматривать не непрерывные функции финансирования, а импульсные инвестиции. Будем рассматривать финансирование в заданные моменты времени

$$
0<t_{1}<t_{2}<\ldots<t_{N} .
$$

В каждый из моментов $t_{k}$ уровень финансирования мы будем обозначать через $u_{k}$. Используя формализм $\delta$ - функций, функцию импульсных инвестиций можно записать следующим образом (2):

$$
u(t)=\sum_{k=1}^{N} u_{k} \delta\left(t-t_{k}\right)
$$



ние (3)

Подставляя эту функцию в интегральное уравнение (1), получаем следующее уравне-

$$
I(t)=\sum_{k=1}^{N} K\left(t-t_{k}\right) u_{k} .
$$

Пусть мы имеем желаемый уровень результативности нашего предприятия, который выражается в виде функции $J(t)$. Тогда задача сводится к нахождению таких значений

$$
u_{1}^{*}, u_{2}^{*}, \ldots, u_{N}^{*},
$$

которые при подстановке в равенство (2) приведут к тождеству (4):

$$
J(t)=\sum_{k=1}^{N} K\left(t-t_{k}\right) u_{k}^{*} .
$$

С математической точки зрения, эта задача является некорректной, поскольку она может не иметь точного решения, может иметь не единственное решение, а также она может быть неустойчивой по отношению к возмущениям.

\section{Метод расчета плана инвестиций}

Планом инвестиций мы будем называть величины:

$$
u_{1}, u_{2}, \ldots, u_{N} \text {. }
$$

Оптимизировать план инвестиций мы будем таким образом, чтобы минимизировать следующую величину (5):

$$
D\left(t, u_{1}, u_{2}, \ldots, u_{N}\right)=\left(\int_{0}^{T}\left|I(t)-J\left(t, u_{1}, u_{2}, \ldots, u_{N}\right)\right|^{2} d t\right)^{\frac{1}{2}} \rightarrow \min .
$$

Таким образом, мы имеем задачу минимизации функции $N$ переменных.

Как мы уже отмечали, эта задача является некорректной, поэтому для численных расчетов необходимо использовать регуляризацию по Тихонову [4]. Для этого мы модифицируем исходную задачу, добавляя к функции $D$ новую функцию с малым параметром (6):

$$
D\left(t, u_{1}, u_{2}, \ldots, u_{N}\right)+\alpha \Omega\left(u_{1}, u_{2}, \ldots, u_{N}\right) \rightarrow \min .
$$

Здесь $\alpha>0$ - это малый параметр, а функция $\Omega$ называется стабилизатором, которая вычисляется по следующей формуле (7):

$$
\Omega\left(u_{1}, u_{2}, \ldots, u_{N}\right)=\sum_{k=1}^{N} u_{k}^{2} .
$$

Использование стабилизатора позволяет регуляризовать исходную задачу.

Для численного нахождения оптимального плана инвестиций предполагается использовать метод роения частиц, который оказывается эффективным при многомерной оптимизации для негладких функций [5].

\section{Результаты численного моделирования}

Рассмотрим модельный пример оптимизации импульсных инвестиций. Мы будем рассматривать финансирование при $N=10$. Будем рассматривать ситуацию, когда нам необходимо обеспечить результативность:

в безразмерных величинах.

$$
I(t)=10
$$

В качестве переходной функции возьмем следующую функцию (8):

$$
K(t)=\frac{t}{1+t^{2}} .
$$


Легко видеть, что эта функция удовлетворяет необходимым условиям. Кроме того, эта функция хорошо отражает экономический эффект от импульсных инвестиций.

Приведем полученные результаты. Оптимальный план импульсных инвестиций приведен на рис. 1. График результативности приведен на рис. 2. Можно видеть, что предложенный план импульсных инвестиций быстро приводит результативность до нужных значений, после чего поддерживает нужный режим результативности.

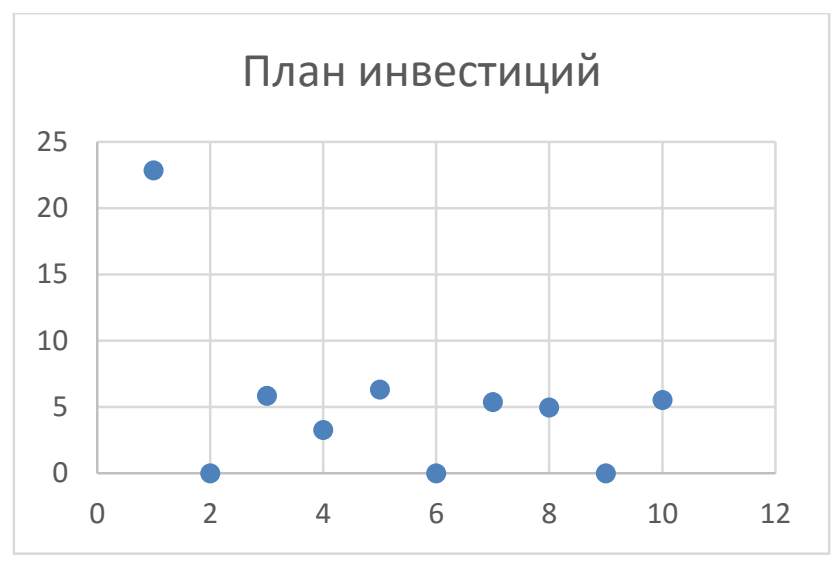

Рис. 1. Оптимальный план импульсных инвестиций

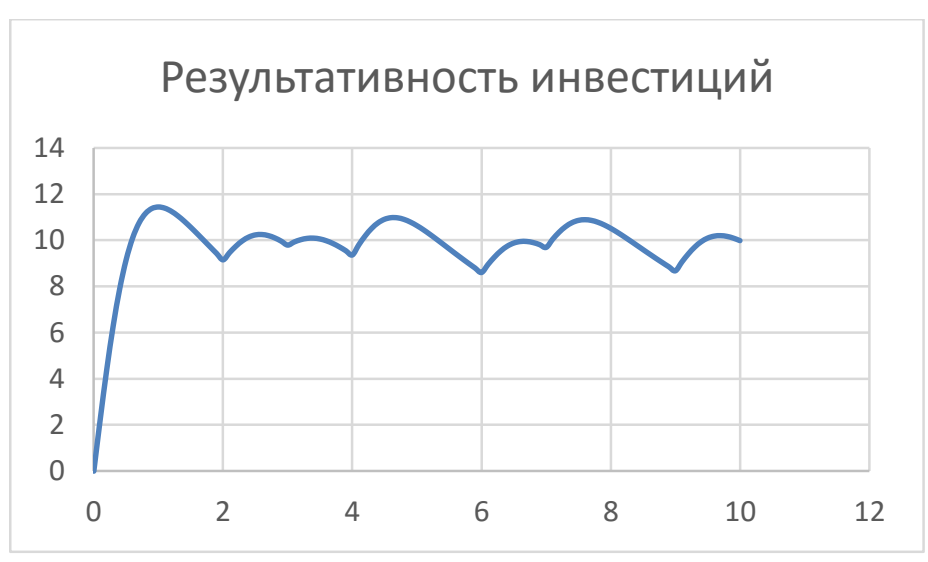

Рис. 2. Результативность оптимальных инвестиций

\section{Заключение}

В работе предложена оригинальная экономико-математическая модель импульсных инвестиций наукоемких предприятий. С помощью этой модели сформулирована экстремальная оптимизационная задача для описания оптимальной схемы импульсных инвестиций с целью поддержания заданного уровня результативности предприятия.

Показано, что полученная экстремальная задача является некорректной по Тихонову, поэтому для построения вычислительных процедур необходимо использовать регуляризацию некорректных экстремальных задач по Тихонову.

Приведен модельный пример расчета оптимальной схемы импульсного финансирования, который демонстрирует рассмотренную экономико-математическую модель. 


\title{
Библиографический список
}

1. Чурсин, А.А. Инвестиции и инновации и их роль в повышении конкурентоспособности организаций / А.А. Чурсин, Р.В. Шамин // Оборонный комплекс - научно-техническому прогрессу России. - 2011. - Вып. 2. - С. 83-87.

2. Чурсин, А.А. Методика количественной оценки конкурентоспособности наукоемкой промышленности / А.А. Чурсин, Р.В. Шамин, Т.В. Кокуйцева // Экономика и управление в машиностроении. 2012. - № 3. - С. 41-47.

3. Васильев, Ф.П. Методы решения экстремальных задач / Ф.П. Васильев. - М.: Наука, 1981. - 552 с.

4. Тихонов, А.Н. Методы решения некорректных задач / А.Н. Тихонов, В.Я. Арсенин. - М.: Наука, $1979,-285$ c.

5. Gurevich, P.L. Reaction-diffusion equations with spatially distributed hysteresis / P.L. Gurevich, R.V. Shamin, S.B. Tikhomirov // SIAM J. Math. Anal. - 2013. - V. 45. - №. 3. - P. 1328-1355.

Дата поступления

в редакичию: 17.04.2019

\section{R.V.Shamin ${ }^{1,2}$, R.A. Chursin ${ }^{2}$, A.G. Shmeleva ${ }^{1,2}$ E.A. Galemina ${ }^{1}$, S.Yu. Obydennova ${ }^{3}$, E.V. Frolagina ${ }^{3}$ \\ THE OPTIMIZATION OF KNOWLEDGE-INTENSIVE ENTERPRISES IMPULSE INVESTMENTS}

\author{
MIREA - Russian technological university ${ }^{1}$ \\ Russian University of peoples' friendship ${ }^{2}$ \\ Nizhny Novgorod State Technical University n.a. R.E. Alekseev ${ }^{3}$
}

Purpose: Simulation model aimed to describe knowledge-intensive enterprises impulse investments is considered.

Design/methodology/approach: The problem is formulated within an original simulation model while numerical results are obtained with particle swarming method.

Findings: The proposed concept allows to estimate impulse investments.

Research limitations/implications: The obtained extremum problem is incorrect according to Tikhonov, therefore, to construct computational procedures, according to Tikhonov, it is necessary to use regularization for ill-posed extremal problems.

Originality/value: New quantitative calculations considering investment plan target indicators achieving optimization problem is proposed. There are presented numerical modeling results for the proposed economic-mathematical model.

Key words: simulation model, investments, knowledge-intensive enterprises, optimization problem, regularization of ill-posed extremal problems. 